\title{
An inconvenient truth: The low r-process fraction in the metal-poor subgiant star HD 140283
}

\author{
A. J. Gallagher* \\ Centre for Astrophysics Research, University of Hertfordshire, United Kingdom \\ E-mail: a.gallagher@herts.ac.uk
}

\section{S. G. Ryan ${ }^{\mathrm{a}}$, A. E. García Pérez ${ }^{\mathrm{b}}$, W. Aoki ${ }^{\mathrm{c}}$}

${ }^{a}$ Centre for Astrophysics Research, University of Hertfordshire, United Kingdom

${ }^{\mathrm{b}}$ Department of Astronomy, University of Virginia, United States

${ }^{\mathrm{c}}$ National Astronomical Observatory, Tokyo, Japan

Current theory regarding heavy element nucleosynthesis in metal-poor environments states that the $r$-process would be dominant. The star HD 140283 has been the subject of debate after it appeared in some studies to be dominated by the $s$-process. We provide an independent measure of the Ba isotope mixture using an extremely high quality spectrum and an extensive $\chi^{2}$ analysis. We have acquired a very high resolution $(R \equiv \lambda / \Delta \lambda=95000)$, very high signal-to-noise $(S / N=1110$ around $4554 \AA$, as calculated in IRAF) spectrum of HD 140283. We exploit hyperfine splitting of the Ba II $4554 \AA$ and $4934 \AA$ resonance lines in an effort to constrain the isotope ratio in 1D LTE. Using the code ATLAs in conjunction with KURUCZ06 model atmospheres we analyse 93 Fe lines to determine the star's macroturbulence. With this information we construct a grid of Ba synthetic spectra and, using a $\chi^{2}$ code, fit these to our observed data to determine the isotopic ratio, $f_{\text {odd }}$, which represents the ratio of odd to even isotopes. The odd isotopes and ${ }^{138} \mathrm{Ba}$ are synthesized by the $r$ - and $s$-process while the other even isotopes $\left({ }^{134,136} \mathrm{Ba}\right)$ are synthesized purely by the $s$-process. We also analyse the Eu lines. We set a new upper limit of the rotation of HD 140283 at $v \sin i \leq 3.9 \mathrm{~km} \mathrm{~s}^{-1}$, a new upper limit on $[\mathrm{Eu} / \mathrm{H}]<-2.80$ and abundances $[\mathrm{Fe} / \mathrm{H}]=-2.59 \pm$ $0.09,[\mathrm{Ba} / \mathrm{H}]=-3.46 \pm 0.11$. This leads to a new lower limit on $[\mathrm{Ba} / \mathrm{Eu}]>-0.66$. We find that, in the framework of a 1D LTE analysis, the isotopic ratios of Ba in HD 140283 indicate $f_{\text {odd }}=0.02 \pm 0.06$, a purely $s$-process signature. This implies that observations and analysis do not validate currently accepted theory. We speculate that a $1 \mathrm{D}$ code, due to simplifying assumptions, is not adequate when dealing with observations with high levels of resolution and signal-to-noise because of the turbulent motions associated with a 3D stellar atmosphere. New approaches to analysing isotopic ratios, in particular 3D hydrodynamics, need to be considered when dealing with the levels of detail required to properly determine them. However published 3D results exacerbate the disagreement between theory and observation.

11th Symposium on Nuclei in the Cosmos

19-23 July 2010

Heidelberg, Germany.

\footnotetext{
* Speaker.
} 


\section{Introduction}

The relative importance of the $r$ - and s-process throughout Galactic history depends on the evolutionary timescales of the proposed sites and their elemental composition. The lifetimes for massive stars are much shorter than for low- to intermediate-mass stars. As such, the interstellar medium (ISM) at the time at which metal-poor (halo) stars were forming ( $\sim 12 \mathrm{Gyr}$ ago) should have been enriched by the supernovae of massive stars and hence, $r$-process material.

A consideration of the possible sites and seed requirements for neutron-capture led Truran [1] to postulate that neutron-capture-element abundances in metal-poor stars should be dominated by those synthesized through the $r$-process. This expectation arises from the realisation that massive stars are capable of producing both the Fe-peak seed nuclei and the high neutron fluxes even from very low-metallicity gas, whereas intermediate-mass stars, while capable of producing neutrons, cannot produce the Fe-peak seeds necessary for the main $s$-process.

Different mixtures of odd and even Ba isotopes are produced by the $r$ - and $s$-process. In particular, ${ }^{134} \mathrm{Ba}$ and ${ }^{136} \mathrm{Ba}$ are produced only by the $s$-process due to shielding by ${ }^{134} \mathrm{Xe}$ and ${ }^{136} \mathrm{Xe}$ in the $r$-process. The line profiles of Ba II $4554 \AA$ and $4934 \AA$ are dependent on the contributions of the $s$ - and $r$-process. A schematic of how the odd and even isotopes align can be seen in Fig. 1. Hyperfine structure (hfs) has been well documented in $\mathrm{Ba}[2,3,4,5]$. As Ba has an even $Z$, only the two odd isotopes, ${ }^{135,137} \mathrm{Ba}$, experience hfs. [6] calculate that the fraction of odd isotopes ${ }^{1}$ of $\mathrm{Ba}$ is $f_{\text {odd }, s}=0.11 \pm 0.01$ for a pure $s$-process mixture of $\mathrm{Ba}$ and infer $f_{\text {odd }, r}=0.46 \pm 0.06$ for a pure $r$-process mixture.

Magain [7] attempted to verify Truran's proposal by measuring the odd fraction of barium isotopes in HD 140283, a well studied metal-poor subgiant at $[\mathrm{Fe} / \mathrm{H}]=-2.5$ [8], but found instead that theory and observations were not compatible. Magain reported the fractional odd isotope ratio, $f_{\text {odd }}$, of $\mathrm{Ba}$ to be $0.08 \pm 0.06$, implying that Ba production in HD 140283 is predominantly due to the $s$-process. The star was later reanalysed by Lambert \& Allende-Prieto [9]. They found a value for $f_{\text {odd }}=0.30 \pm 0.21$ and concluded that, contrary to Magain's result, the star is $r$-process dominated.

While we conducted our study, Collet et al. [10] reanalysed the [9] spectrum. In 1D LTE they found that $f_{\text {odd }}=0.33 \pm 0.13$. However, their analysis of the line using 3D hydrodynamics gives a value for $f_{\text {odd }}=0.15 \pm 0.12$. This value is once more at odds with the high $r$-process fraction expected under Truran's hypothesis. Against this background, we sought to improve the determination of the $r$-process contribution to $\mathrm{Ba}$ in this star to help us understand the apparent conflicts. We use a very high quality spectrum of $\operatorname{HD} 140283(R \equiv \lambda / \Delta \lambda=95000, S / N=1110$ per $12 \mathrm{~m} \AA$ wide pixel around the Ba II $4554 \AA$ line) taken from the Subaru HDS during its commissioning, to conduct our analysis.

\section{Macroturbulence}

We create a grid of 385 synthetic spectra using ATLAS for 11 values of macroturbulence and 35 values of $A(\mathrm{Fe})$. Each synthetic spectrum covered the wavelength range $4100-6900 \AA$ in intervals of $\Delta \lambda=0.01 \AA$. To determine the best fit for the combination of instrumental broadening and macroturbulence, which we model as a single Gaussian of FWHM $v_{\text {conv }}$, we compare our synthetic

$$
{ }^{1} f_{\text {odd }} \equiv\left[N\left({ }^{135} \mathrm{Ba}\right)+N\left({ }^{137} \mathrm{Ba}\right)\right] / N(\mathrm{Ba})
$$


model grid to the observed spectrum employing a $\chi^{2}$ test, $\chi^{2} \equiv \sum\left(O_{i}-M_{i}\right)^{2} / \sigma_{i}^{2}$, where $O_{i}$ is the observed continuum-normalised profile, $M_{i}$ is the model profile and $\sigma_{i}^{2}$ is the standard deviation of the observed points that define the continuum, i.e. $\sigma=(S / N)^{-1}$. We use $93 \mathrm{Fe}$ I and Fe II lines, which were individually fit using a $\chi^{2}$ code [11]. We found $v_{\text {conv }}=5.75 \pm 0.02 \mathrm{~km} \mathrm{~s}^{-1}$, where the error is represented by the standard error, $\sigma / \sqrt{N}$.

For the purposes of this analysis we assume that the star has no significant rotation. However, during the course of our analysis it was determined that the star's upper limit on rotation, $v \sin i \leq$ $3.89 \pm 0.02 \mathrm{~km} \mathrm{~s}^{-1}$.

\section{The Ba II resonance lines and the barium isotopic ratio}

We use the hfs information from [3] and [5] to compute energy level splittings for the lower and upper levels of ${ }^{135,137} \mathrm{Ba}$ in the $4554 \AA$ and $4934 \AA$ lines, and incorporated the isotopic shifts relative to ${ }^{138} \mathrm{Ba}$. Using the $s$ - and $r$-process contributions to the five isotopes from [6], we construct line lists for pure $s$-process and pure $r$-process isotope mixes for the two Ba II lines. From these two lists we created a further 13 hybrid line lists. These covered a range for $f_{\text {odd }}$ equal to $0.00 \leq f_{\text {odd }} \leq 0.46$ where $\Delta f_{\text {odd }}=0.035$.
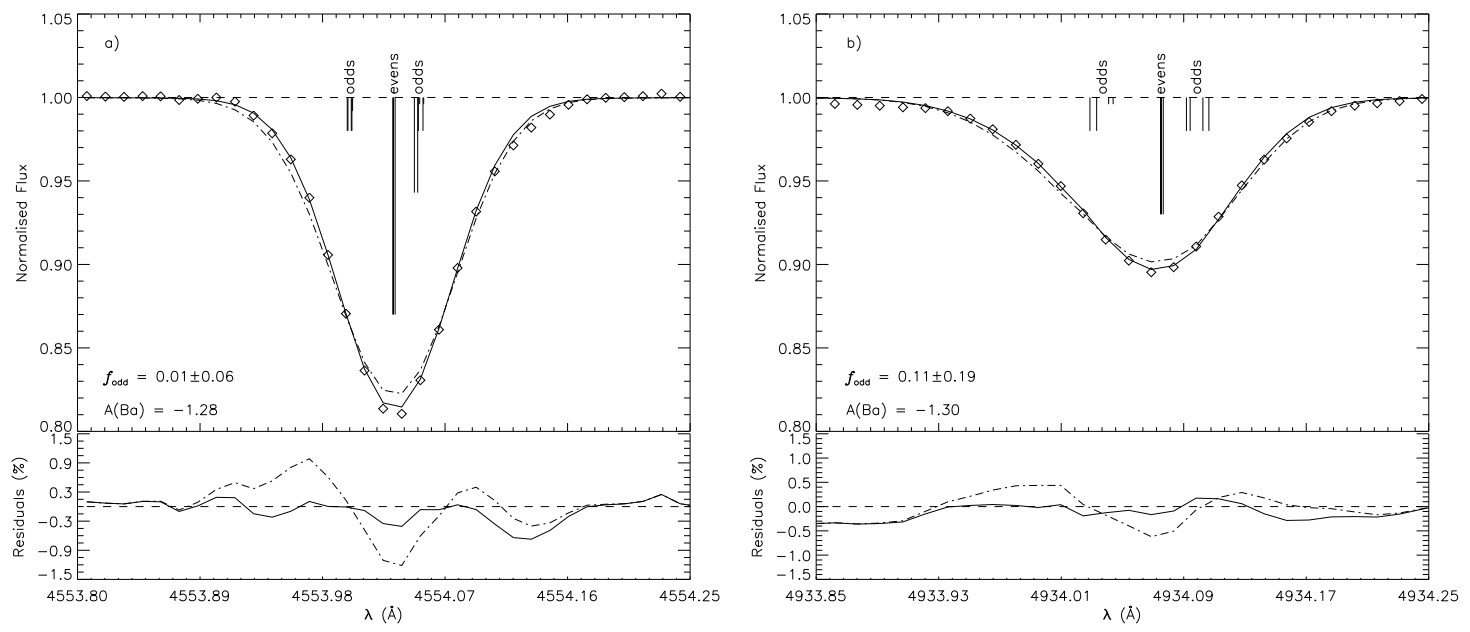

Figure 1: Panel a): The best statistical fit synthetic profile (solid line) for the observed Ba II $4554 \AA$ line (diamonds) with the residual plots below. For comparison, a pure $r$-process line and residual has been plotted (dash-dot line); the value for $A(\mathrm{Ba})$ has been optimised to one that minimises $\chi^{2}$, while values for $A(\mathrm{Fe})$ and macroturbulence remain the same as for the solid line. Panel $b$ ): Shows the same as $a$ ) for the $4934 \AA$ line.

The $\mathrm{Ba}$ abundances of the two lines was found to be $A(\mathrm{Ba})=-1.29 \pm 0.08$. Using solar abundances in [12] we find that for HD 140283, $[\mathrm{Ba} / \mathrm{H}]=-3.46 \pm 0.11$, and hence $[\mathrm{Ba} / \mathrm{Fe}]=$ $-0.87 \pm 0.14$. From the $\chi^{2}$ analysis we find the best statistical fit for the $4554 \AA$ line is $f_{\text {odd }}=$ $0.01 \pm 0.06$. The best statistical fit for the $4934 \AA$ line indicates a value of $f_{\text {odd }}=0.11 \pm 0.19$. These are shown in Fig. 1. Inverse-variance-weighting the results gives an overall result $f_{\text {odd }}=$ $0.02 \pm 0.06$. 


\section{Fe line asymmetries}

We under-fit the red wing of the $4554 \AA$ line between $4554.11 \AA$ and $4554.17 \AA$ (see Fig. 1 (a)). Studies [9] and [10] in 1D, see the same residual feature at this wavelength interval. Reanalysis in 3D [10] appears to remove the feature in the wing. This would suggest that it is a result of convection in a 3D atmosphere, which the simplistic assumptions made in a 1D LTE code cannot replicate.

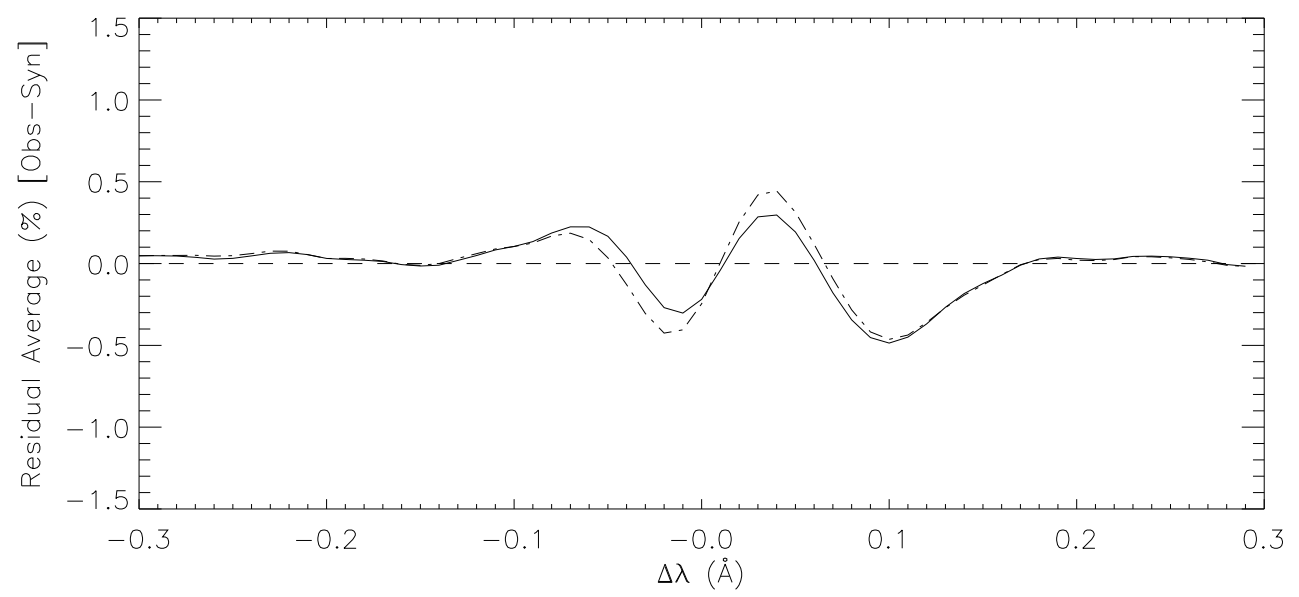

Figure 2: The reduced-noise residual plot for $82 \mathrm{Fe}$ lines without other close absorption features. Solid line: "'optimised"' residuals based on synthetic spectra calculated using the $v_{\text {conv }}$ and $\Delta \lambda$ values calculated for each line. Dash-dot line: "'average"' residuals based on synthetic spectra calculated using the average $\Delta \lambda$ $(-12.04 \mathrm{m \AA})$ and $v_{\text {conv }}\left(5.75 \mathrm{~km} \mathrm{~s}^{-1}\right)$.

To explore this further, we have searched for evidence of asymmetries in the Fe-line data. A reduced-noise plot (Fig. 2) was produced by co-adding the residuals of the Fe lines used to constrain macroturbulence. The average residual plot (dash-dot line) is very asymmetric, in that the blue wings are better fit than the red wings. The large residuals in the red wings, around $60-170 \mathrm{~m} \AA$ from the line centre, are clearly not due to errors in the central wavelengths of the Fe lines, which are known to better than $1 \mathrm{~m} \AA$. The optimised residual plot (solid line) slightly reduces the overall amplitude of the residual. However, even after allowing each line to be optimised, the residuals are still quite asymmetric. From Fig. 2 we can see that a similar red feature to the one we see in Fig. 1 for $\mathrm{Ba}$ is present in both Fe residual plots, and appears at the same distance from the centroid of the Fe lines as the residuals for the Ba $4554 \AA$ line $(\sim 100 \mathrm{~m} \AA)$. This further leads us to believe that this feature may be the result of convection in the observed, dynamic atmosphere, similar to the feature seen in the Ba spectrum, that underlying assumptions in 1D LTE do not compensate for.

\section{Europium abundance limit}

Within our spectral range $(4118-6253 \AA)$ there are two Eu II resonance lines, $4129.70 \AA$ and $4205.05 \AA$. However, these lines do not appear strongly in our spectrum. A detailed study of the two lines used in our analysis can be found in [13]. 
We find that a $[\mathrm{Eu} / \mathrm{H}]$ abundance of -2.80 seems to be a generous upper limit on the Eu abundance, rather than a genuine detection and lower than detections cited elsewhere. Therefore we assign a lower limit $[\mathrm{Ba} / \mathrm{Eu}]>-0.66$. This marginally excludes an $r$-process ratio, whether we assume an $r$-process limit set in [14] or [6]. A pure $s$-process ratio or a mixed $s$ - and $r$-process regime, is compatible with the data.

\section{Conclusions}

For HD 140283 we obtain $[\mathrm{Fe} / \mathrm{H}]=-2.59 \pm 0.09,[\mathrm{Ba} / \mathrm{Fe}]=-0.87 \pm 0.14$, and $[\mathrm{Eu} / \mathrm{H}]<$ -2.80 . Using a $1 \mathrm{D}$ LTE analysis, we find $f_{\text {odd }}=0.02 \pm 0.06$, corresponding to a Ba isotopic fraction which indicates a $100 \%$ contribution by the s-process. This result contradicts the theory put forward by Truran [1]. We have set a new lower limit to the $[\mathrm{Ba} / \mathrm{Eu}]$ ratio, $[\mathrm{Ba} / \mathrm{Eu}]>-0.66$. This lower limit marginally rules out a pure $r$-process ratio in HD 140283, consistent with the isotopic fraction for barium. We set a new limit on the rotation of HD 140283: $v \sin i<3.9 \mathrm{~km} \mathrm{~s}^{-1}$.

By examining the spectral residuals for $82 \mathrm{Fe}$ lines and for Ba $4554,4934 \AA$, we find strong line asymmetries in the red wing. These may show the shortcomings of using a 1D LTE analysis to explore isotope ratios; using a more sophisticated 3D analysis may be warranted. A much more detailed explanation of the all topics discussed here, which includes a thorough error analysis can be found in [13].

\section{References}

[1] Truran, J. W., 1981, A\&A, 97:391.

[2] Rutten, R. J., 1978, Sol. Phys., 56:237.

[3] Wendt, K., Ahmad, S. A., Buchinger, F., et al., 1984, Zeitschrift fur Physik, 318:125.

[4] Cowley, C. R. and Frey, M., 1989, ApJ, 346:1030.

[5] Villemoes, P., Arnesen, A., Heijkenskjold, F., et al., 1993, Journal of Physics B Atomic Molecular Physics, 26:4289.

[6] Arlandini, C., Käppeler, F., Wisshak, K., et al., 1999, ApJ, 525:886.

[7] Magain, P., 1995, A\&A, 297:686.

[8] Aoki, W., Inoue, S., Kawanomoto, S., et al., 2004, A\&A, 428:579.

[9] Lambert, D. L. \& Allende Prieto, C., 2002, MNRAS, 335:325.

[10] Collet, R., Asplund, M., Nissen, P. E., 2009, Publications of the Astronomical Society of Australia, 26:330.

[11] García Pérez, A. E., Aoki, W., Inoue, S., et al., 2009, A\&A, 504:213.

[12] Grevesse, N., Sauval, A. J., 1998, Space Science Reviews, 85:161.

[13] Gallagher, A. J., Ryan, S. G., García Pérez, A. E., et al., 2010, A\&A, in press, [ArXiv: 1008.3541$]$.

[14] Burris, D. L., Pilachowski, C. A., Armandroff, T. E., et al., 2000, ApJ, 544:302. 